\title{
Hardening of smooth pulsed laser deposited PMMA films by heating
}

\author{
Britta Fuchs · Felix Schlenkrich • Susanne Seyffarth • \\ Andreas Meschede · Robert Rotzoll • Philipp Vana • \\ Peter Großmann · Klaus Mann · Hans-Ulrich Krebs
}

Published online: 22 December 2009

(c) The Author(s) 2009. This article is published with open access at Springerlink.com

\begin{abstract}
Smooth poly(methyl methacrylate) (PMMA) films without any droplets were pulsed laser deposited at a wavelength of $248 \mathrm{~nm}$ and a laser fluence of $125 \mathrm{~mJ} / \mathrm{cm}^{2}$. After deposition at room temperature, the films possess low universal hardness of only $3 \mathrm{~N} / \mathrm{mm}^{2}$. Thermal treatments up to $200^{\circ} \mathrm{C}$, either during deposition or afterwards, lead to film hardening up to values of $200 \mathrm{~N} / \mathrm{mm}^{2}$. Using a combination of complementary methods, two main mechanisms could be made responsible for this temperature induced hardening effect well above the glass transition temperature of $102^{\circ} \mathrm{C}$. The first process is induced by the evaporation of chain fragments and low molecular mass material, which are present in the film due to the ablation process, leading to an increase of the average molecular mass and thus to hardening. The second mechanism can be seen in partial cross-linking of the polymer film as soon as chain scission occurs at higher temperatures and the mobility and reactivity of the polymer material is high enough.
\end{abstract}

\footnotetext{
B. Fuchs - F. Schlenkrich - S. Seyffarth - A. Meschede -

H.-U. Krebs ( $\varangle)$

Institut für Materialphysik, University of Göttingen,

Friedrich-Hund-Platz 1, 37077 Göttingen, Germany

e-mail: krebs@ump.gwdg.de

R. Rotzoll · P. Vana

Institut für Physikalische Chemie, University of Göttingen,

Tammannstraße 6, 37077 Göttingen, Germany

P. Großmann · K. Mann

Laser-Laboratorium Göttingen e.V., Hans-Adolf-Krebs-Weg 1,

37077 Göttingen, Germany
}

\section{Introduction}

Thin polymer films are of high interest for many applications, e.g. microelectronics or coatings. Besides many other techniques, pulsed laser deposition (PLD) has been proven to allow the deposition of polymer thin films as first described by Hansen and Robitaille [1, 2]. For poly(methyl methacrylate) (PMMA), at a wavelength of $248 \mathrm{~nm}$ the ablation process from the target is well understood, indicating that during incubation photochemical changes occur within the PMMA target [3-7]. A large amount of $\mathrm{CO}, \mathrm{CO}_{2}$ and methyl formate, $\mathrm{HCOOCH}_{3}$, was observed, clearly indicating side chain scissions of methyl ester $* \mathrm{COOCH}_{3}$. This is directly connected with the formation of $\mathrm{C}=\mathrm{C}$ double bonds within the main chain, inducing an increase of UV absorption close to the target surface. Thus, ablation of modified PMMA and small fractions takes place, which are then deposited on the substrate. The mechanisms of film growth on the substrate surface are still a matter of discussion $[6,8,9]$. In the literature, it was claimed that smooth films can only be obtained for strong absorbing materials [1, 2]. But in an earlier work, we already showed that for PMMA completely smooth films (with a surface roughness of less than $1 \mathrm{~nm}$ ) can be grown without any droplets by PLD in a narrow fluence range slightly above the deposition threshold [10] also when using a laser wavelength of $248 \mathrm{~nm}$, where the absorption of the laser radiation is only $500 \mathrm{~cm}^{-1}$ [11].

In this paper, we will show that these smooth laser deposited polymer films have low hardness after deposition, but can easily be hardened by heating them during deposition or afterwards to temperatures well above the glass transition temperature. Mechanisms responsible for this hardening effect at elevated temperatures are investigated by a combination of complementary methods. 


\section{Experimental details}

For these experiments, a standard pulsed laser deposition setup was used [12]. An excimer laser beam (Lambda Physik LPX110i, KrF, $248 \mathrm{~nm}, 30 \mathrm{~ns}$ ) is focused onto targets consisting of dense, hot pressed and transparent PMMA (Aldrich, $M_{\mathrm{w}}=996 \mathrm{~kg} / \mathrm{mol}$ ) [13]. The background pressure within the deposition chamber was lower than $5 \times 10^{-8}$ mbar. All PMMA films investigated in this study were deposited using a laser fluence of about $125 \mathrm{~mJ} / \mathrm{cm}^{2}$ and were smooth without any droplets as described in [10]. Films of thicknesses between $100 \mathrm{~nm}$ and $30 \mu \mathrm{m}$ were grown at different substrate temperatures. Most films were deposited on $\mathrm{Si}(111)$ substrates, but for the Fourier transform infrared (FT-IR) measurements (using a Bruker Tensor 37 in reflection mode) rough $\mathrm{Al}$ substrates were taken. Molecular weight distributions were determined by size exclusion chromatography (SEC). For this purpose, a Waters system was used with 3 PSS SDV columns with $5 \mu \mathrm{m}$ particle size and pore sizes of $10^{4}, 10^{2}$, and $10 \mathrm{~nm}$. Differential thermal analysis (DTA) and thermogravimetric analysis (TGA) in combination with mass spectroscopy were performed using a Netsch STA 449 Jupiter with a heating rate of $1 \mathrm{~K} / \mathrm{min}$. The film hardness was determined by a Fischer Nanoscope 100C XYm applying an indentation force of $1 \mathrm{mN}$ in $20 \mathrm{~s}$. In order to study the chemical environment of the C-atoms, near edge X-ray absorption spectroscopy (NEXAFS) was performed at the carbon K-edge (photon energies from 280$310 \mathrm{eV}$ ), using a table-top laser-driven XUV plasma source (details described earlier in [14]).

\section{Results and discussion}

The temperature dependence of the universal hardness of laser deposited PMMA films was studied up to temperatures of $200^{\circ} \mathrm{C}$. For these experiments, films with thicknesses of up to $30 \mu \mathrm{m}$ were grown in order to avoid any influence of the substrate on the hardness measurements. The polymer films were grown either at different substrate temperatures or were deposited at room temperature and annealed afterwards. In Fig. 1, it can clearly be seen that the universal hardness HU cursive of PMMA films deposited at room temperature is strongly reduced (to about $3 \mathrm{~N} / \mathrm{mm}^{2}$ ) in comparison to values of the PMMA targets (about $290 \mathrm{~N} / \mathrm{mm}^{2}$ ). Additionally, the film is more duroplastic while the target shows a total elastic behavior. At increased substrate temperature as well as at raised annealing temperatures, the films start to harden above temperatures of about $60^{\circ} \mathrm{C}$, reaching values of up to about $200 \mathrm{~N} / \mathrm{mm}^{2}$ at $200^{\circ} \mathrm{C}$. It is interesting to note that this hardening is independent of whether the films were deposited at elevated substrate temperatures or were annealed after deposition at room temperature. Thus, the effect of film growth seems to be marginal.

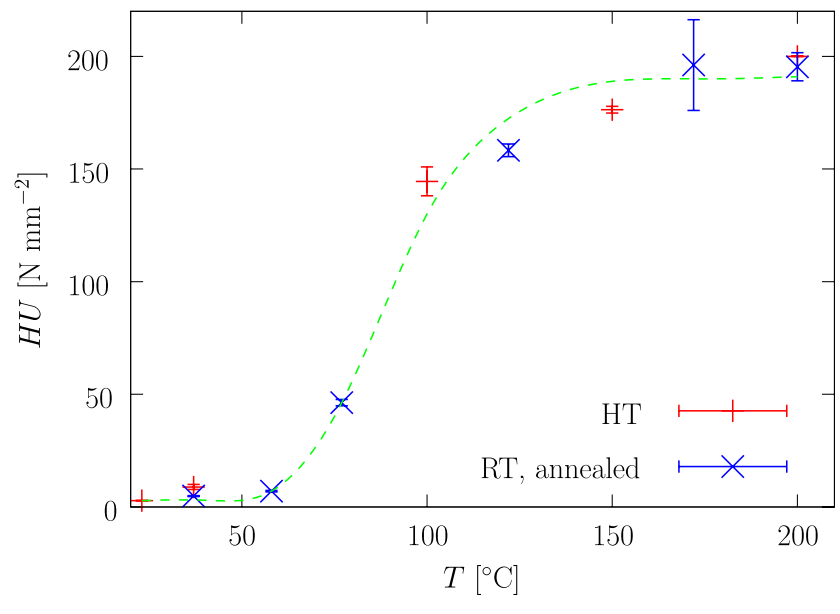

Fig. 1 Hardness measurements for films deposited/annealed at temperatures up to $200^{\circ} \mathrm{C}$. A strong increase of the universal hardness near the glass transition temperature is observed independently of whether the polymer is heated during deposition or afterwards

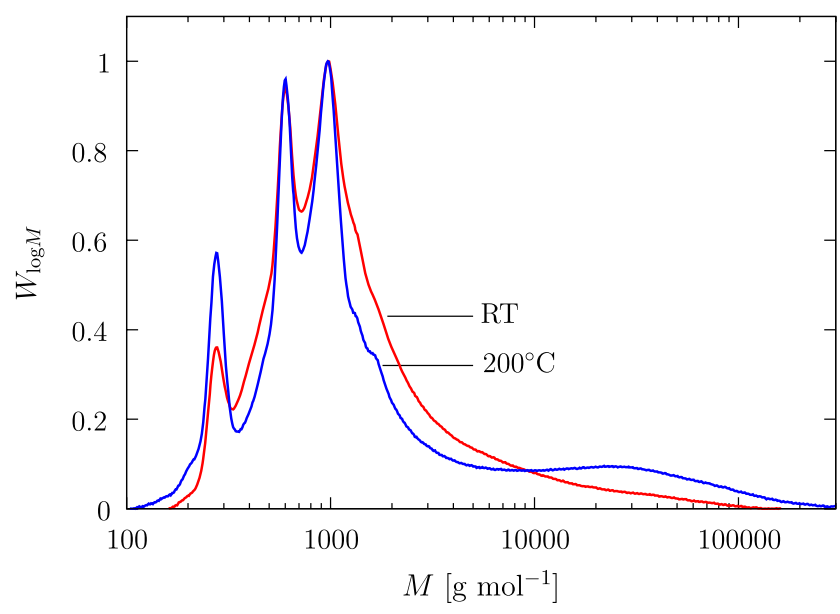

Fig. 2 SEC measurements of PMMA films laser deposited at room temperature and $200^{\circ} \mathrm{C}$, respectively. For films grown at room temperature the spectra show a strong reduction of the average molar mass to $2,900 \mathrm{~g} / \mathrm{mol}$ which increases at $200^{\circ} \mathrm{C}$ to $7,400 \mathrm{~g} / \mathrm{mol}$

Detailed investigations were performed to understand the mechanisms leading to the strongly reduced hardness for the polymer films deposited at room temperatures and to the hardening during temperature treatments.

To analyze the molecular mass distribution within the films, SEC measurements were performed. In Fig. 2, the SEC spectra normalized to the highest peak intensity are depicted. The polymers grown at room temperature exhibit a reduced average molar mass $M_{\mathrm{w}}$ of about $2,900 \mathrm{~g} / \mathrm{mol}$, and thus the chain length of the polymer in the film is strongly reduced compared to the starting material $(996,000 \mathrm{~g} / \mathrm{mol})$. It can also clearly be noticed that a substantial amount of oligomers exists in the PMMA films. This is a clear indication that main chain scission has taken place during ablation. These shorten chains are most likely responsible for 


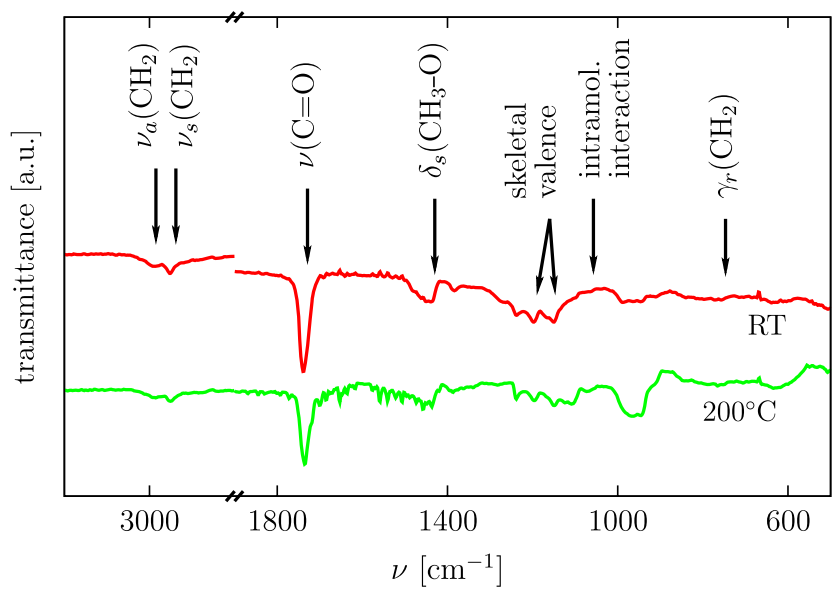

Fig. 3 FT-IR measurements of PMMA films laser deposited at room temperature and $200^{\circ} \mathrm{C}$ showing an intact chemical structure

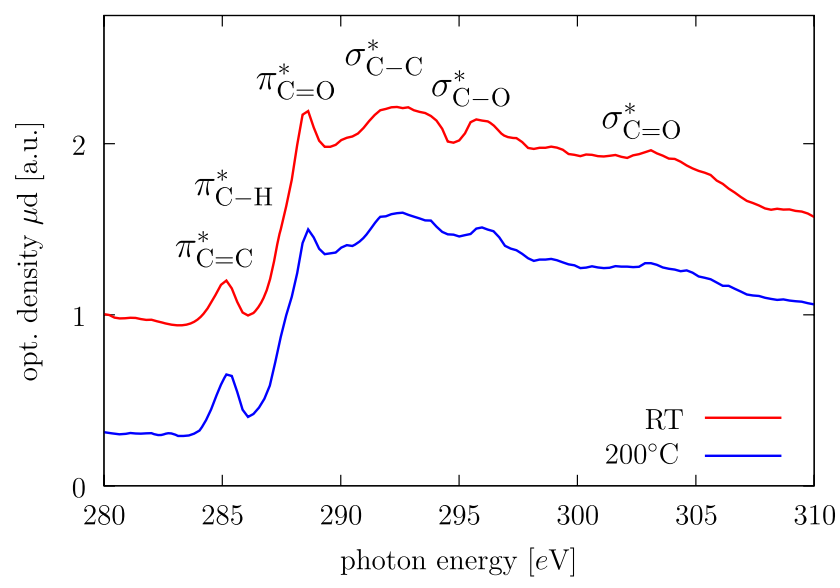

Fig. 4 NEXAFS measurements at the C-edge for PMMA films deposited at room temperature and $200^{\circ} \mathrm{C}$, respectively. Note the peak of the $1 \mathrm{~s} \rightarrow \pi_{\mathrm{C}=\mathrm{C}}^{*}$ transition due to incubation processes and its increase for higher temperature, whereas the peak of the $1 \mathrm{~s} \rightarrow \pi_{\mathrm{C}=\mathrm{O}}^{*}$ transition slightly decreases at $200^{\circ} \mathrm{C}$

the softness of the deposited polymer film. After deposition at a substrate temperature of $200^{\circ} \mathrm{C}$, the average molecular weight increases to about $7,400 \mathrm{~g} / \mathrm{mol}$. This mass increase is a first hint for a preferential evaporation of the short-length material and an increase of cross-linking due to radical formation in the polymer.

The chemical composition of the grown film was studied by FT-IR. The FT-IR spectra of the films deposited at room temperature show all the functional groups of PMMA, indicating that a high amount of deposited PMMA still has intact composition (see Fig. 3). Even when depositing PMMA at elevated substrate temperature of $200^{\circ} \mathrm{C}$, the chemical composition remains almost the same.

In order to study the local environment of the $\mathrm{C}$-atoms in more detail, NEXAFS measurements were performed at the carbon K-absorption edge. Figure 4 shows a comparison of NEXAFS spectra for samples deposited at room temper-

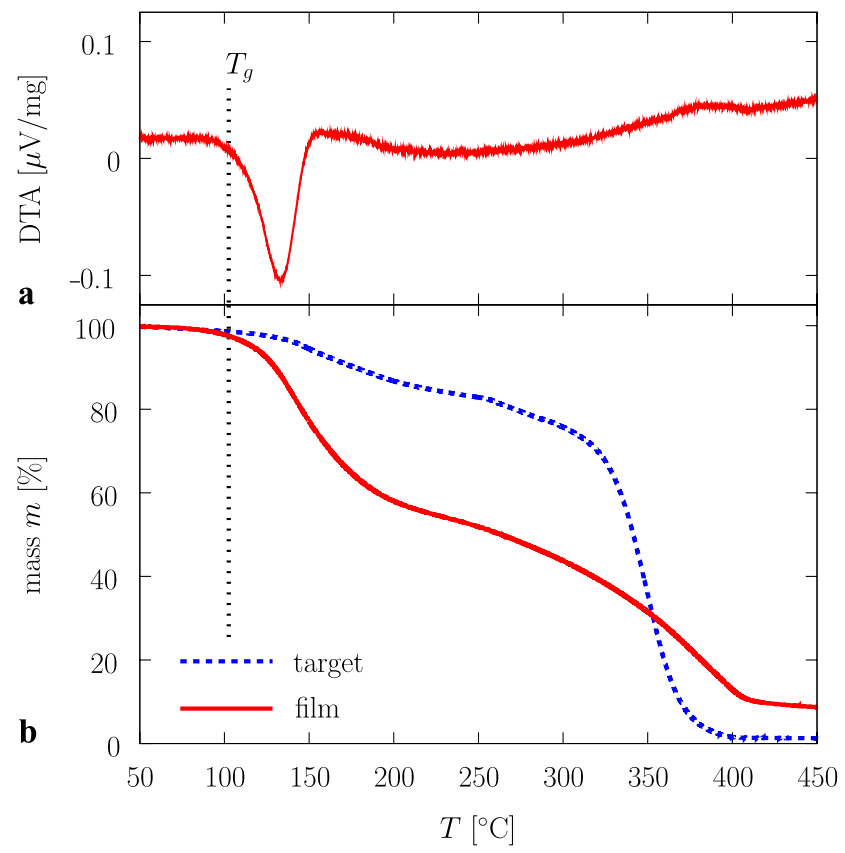

Fig. 5 In (a), the DTA measurement of a laser deposited PMMA film is shown with a glass transition temperature at $102^{\circ} \mathrm{C}$. In (b), the TGA measurement for the same film and the target material is depicted indicating that the material loss occurs in two steps, one above the glass transition temperature and the second one at high temperatures when the polymer degrades

ature and at $200^{\circ} \mathrm{C}$, respectively. In both cases, the spectra show all the absorption maxima and features typically for PMMA bulk material (see, for instance, [15]). However, two main differences become visible. First, the intensity of the sharp peak of the $1 \mathrm{~s} \rightarrow \pi_{\mathrm{C}=\mathrm{O}}^{*}$ transition at $288.5 \mathrm{eV}$, which is highest in bulk PMMA, is reduced compared to literature $[15,16]$. Second, a peak of the $1 \mathrm{~s} \rightarrow \pi_{\mathrm{C}=\mathrm{C}}^{*}$ transition at $285 \mathrm{eV}$ exists, which does not occur in bulk material. Both differences can be explained by the incubation processes at the target surface $[3-7,17,18]$. They can be explained by the loss of the whole or parts of the methyl ester side groups and the formation of $\mathrm{C}=\mathrm{C}$ double bonds. After deposition at $200^{\circ} \mathrm{C}$, only small changes are observed in the NEXAFS spectra. Mainly a slight increase of the $1 \mathrm{~s} \rightarrow \pi_{\mathrm{C}=\mathrm{C}}^{*}$ peak and a small reduction of the $1 \mathrm{~s} \rightarrow \pi_{\mathrm{C}=\mathrm{O}}^{*}$ peak is observed, both indicating an evaporation of ester groups.

The glass transition temperature $T_{\mathrm{g}}$ and the mass loss during heating were determined by DTA and TGA measurements. For these experiments, PMMA films deposited at room temperature were dissolved from the substrate and post-annealed (Fig. 5(a)). These experiments show that the glass transition temperature of the polymer film is only slightly reduced to about $102^{\circ} \mathrm{C}$ compared to bulk PMMA $\left(113^{\circ} \mathrm{C}\right)$. This can be attributed to the observed slight changes and reduced chain length of the PMMA film [19]. In Fig. 5(b), TGA-measurements are shown for a PMMA target and a film. With increasing annealing temperature, 


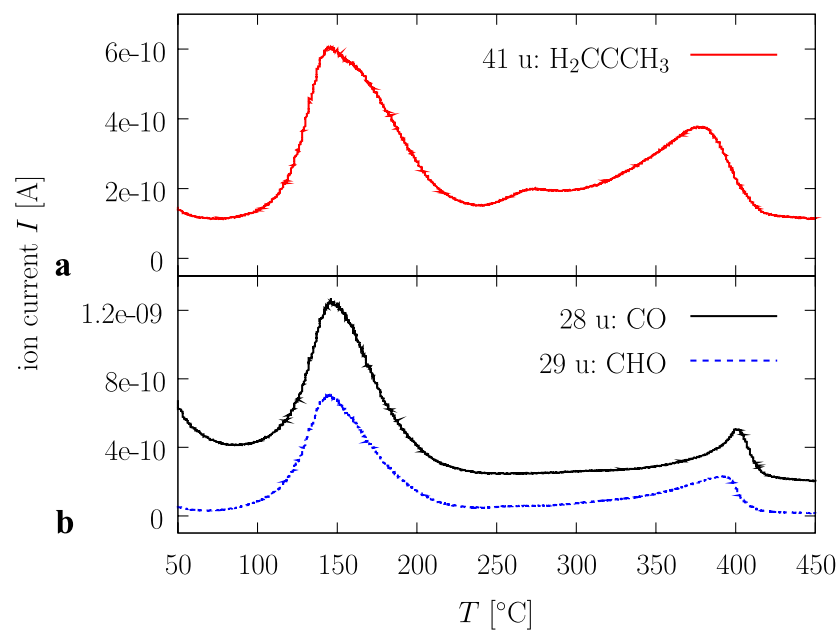

Fig. 6 Mass spectrometry measurements performed during heating show above the glass transition temperature the preferred evaporation of (a) the monomer part without the ester group and (b) the carbonyl group $\mathrm{CO}$ and the aldehyde group $\mathrm{CHO}$. Together with molecular hydrogen $\mathrm{H}_{2}$ these build the complete monomer MMA

the masses of film and target both decrease in two steps, but with different step heights. The first decrease occurs above the glass transition temperature. Here the film looses about $40 \%$ of its mass, whereas only $10 \%$ of the bulk material evaporates. This is an indication for an evaporation of shorter fragments, which occur with a higher amount in the polymer film than in the target because of the fragments resulting from the ablation process. For higher temperatures above $250^{\circ} \mathrm{C}$, the mass of the polymer film starts to vanish due to degradation and subsequent evaporation. Due to the reduced chain length, the degradation of the polymer film starts at lower temperatures compared to the bulk material. In addition, the degradation of the target material occurs in a smaller temperature range because of the lower polydispersity $D=M_{\mathrm{w}} / M_{\mathrm{n}}=3.4$ (with the number average molar mass $\left.M_{\mathrm{n}}\right)$. For the PMMA film the polydispersity is up to $D=5.0$ for room temperature conditions and increases even up to $D=9.5$ for films deposited at $200^{\circ} \mathrm{C}$ because of the higher amount of longer chains due to cross-linking.

The evaporation of small polymer fragments during annealing was confirmed by mass spectroscopy. Over the whole investigated temperature range (up to $450^{\circ} \mathrm{C}$ ) fragments with mass units $\mathrm{u}$ of 2 (interpreted as $\mathrm{H}_{2}$ ) and 39 (interpreted as $\mathrm{H}_{2} \mathrm{CCCH}$ ) were observed with high intensities. Besides those, mainly three distinguished masses with mass units of 28, 29 and 41 were detected at temperatures close to the glass transition temperature (see Fig. 6). Two peaks occur in the mass spectra which correspond to the two steps already seen in the TGA measurement. They clearly indicate that the monomer part without the ester group $\left(\mathrm{H}_{2} \mathrm{CCCH}_{3}\right)$ as well as two constituents of the ester group, i.e. the carbonyl group (CO) and the aldehyde group ( $\mathrm{CHO})$, show an intensified evaporation above the glass transition temperature and during degradation of the polymer at high temperatures. But these three masses are a leading contribution only for the more interesting first peak. A more detailed analysis of 16 further selected masses (partially measured also in $[6,20]$ ) indicate that the second peak above $250^{\circ} \mathrm{C}$ is dominated by a series of small fragments typically for a total degradation of the polymer film.

\section{Conclusions}

In summary, from our experiments we conclude that two types of very smooth PMMA films can be prepared by pulsed laser deposition at $248 \mathrm{~nm}$ slightly above the deposition threshold. On the one hand, very soft PMMA films are obtained when depositing the material at room temperature. The strongly reduced hardness of the PMMA films is caused by the reduced chain lengths of the polymers and the occurrence of polymer fragments after deposition. NEXAFS spectra indicate the appearance of $\mathrm{C}=\mathrm{C}$ double bonds in the films originating from incubation processes at the target. Due to the polymer fragments deposited on the substrate, the film is more duroplastic while the target shows a totally elastic behavior. On the other hand, films with increased hardness, which could be more interesting for applications, can be grown at temperatures well above the glass transition temperature by simply heating them either during or after deposition. Then the mobility within the polymer film is high enough that small fragments also from inner parts of the film can reach the surface to evaporate, and simultaneously, chain scissions occur, leading to a cross-linking within the polymer.

Acknowledgements We like to thank Katrin Gehrke for her help with the combined DTA, TGA and mass spectroscopy measurement. This work was supported by the Deutsche Forschungsgemeinschaft (SFB 602 and SFB 755) as well as Graduiertenkolleg 782.

Open Access This article is distributed under the terms of the Creative Commons Attribution Noncommercial License which permits any noncommercial use, distribution, and reproduction in any medium, provided the original author(s) and source are credited.

\section{References}

1. S.G. Hansen, T.E. Robitaille, Appl. Phys. Lett. 52, 81 (1988)

2. S.G. Hansen, T.E. Robitaille, J. Appl. Phys. 64, 2122 (1988)

3. R.C. Estler, N.S. Nogar, Appl. Phys. Lett. 49, 1175 (1986)

4. R. Larciprete, M. Stuke, Appl. Phys. B 42, 181 (1987)

5. S. Küper, M. Stuke, Appl. Phys. A 49, 211 (1989)

6. G.B. Blanchet, Macromolecules 28, 4603 (1995)

7. D.B. Chrisey, A. Piqué, R.A. McGill, J.S. Horwitz, B.R. Ringeisen, Chem. Rev. 103, 553 (2003)

8. S.W. Wee, S.M. Park, Bull. Korean Chem. 22, 914 (2001) 
9. Y. Tsuboi, H. Adachi, E. Yamamoto, A. Itaya, Jpn. J. Appl. Phys. 41, 885 (2002)

10. B. Lösekrug, A. Meschede, H.U. Krebs, Appl. Surf. Sci 254, 1312 (2007)

11. R. Srinivasan, B. Braren, K.G. Casey, Pure Appl. Chem. 62, 1581 (1990)

12. H.U. Krebs, O. Bremert, Appl. Phys. Lett. 62, 2341 (1993)

13. E. Süske, T. Scharf, H.U. Krebs, E. Panchenko, T. Junkers, M. Egorov, M. Buback, H. Kijewski, J. Appl. Phys. 97, 063501 (2005)

14. C. Peth, F. Barkusky, K. Mann, J. Phys. D: Appl. Phys. 41, 105202 (2008)
15. J. Stöhr, in NEXAFS Spectroscopy, ed. by R. Gromer, Springer Series in Surface Sciences, vol. 25 (Springer, Berlin, 1992)

16. O. Dhez, H. Ade, S.G. Urquhart, J. Electron. Spectrosc. Relat. Phenom. 128, 85-96 (2003)

17. E. Sutcliffe, R. Srinivasan, J. Appl. Phys. 60, 3315 (1986)

18. G.B. Blanchet, P. Cotts, C.R. Fischer, J. Appl. Phys. 88, 2975 (2000)

19. K. O’Driscoll, R.A. Sanayeu, Macromolecules 24, 4479 (1991)

20. A. Gupta, R. Liang, F.D. Tsay, J. Moacanin, Macromolecules 13, $1696(1980)$ 